\title{
Prevalence of Orofacial Dysfunctions And Oral Habits in A Sample of 4-5-Year Old Bulgarian Children
}

\author{
Keti Yovcheva ${ }^{1}$,Miroslava Yordanova ${ }^{1}$, Svetlana Yordanova ${ }^{1}$,Nina Musurlieva ${ }^{2}$ \\ ${ }^{l}$ (Department Of Orthodontics, Faculty Of Dental Medicine, Medical University - Plovdiv, Bulgaria) \\ ${ }^{2}$ (Department Of Social Medicine And Public Health, Faculty Of Public Health, Medical University - Plovdiv,
} Bulgaria)

\begin{abstract}
The aim of this study is to estimate the prevalence of orofacial dysfunctions in a sample of Bulgarian kindergarten children with primary dentition. 471 girls and boys participated in this observational crosssectional epidemiological study. The presence of non-nutrive sucking behaviors, open mouth posture, nasal breathing impairment, swallowing pattern and symptoms of speech disorders were assessed and analyzed. $31 \%$ of the examined children have a harmful sucking habit. An open mouth posture was observed in $46.1 \%$ of all children, while impaired nasal breathing was assessed in $19.6 \%$ of the subjects. Infantile swallowing was preserved in $37 \%$ of all participants and $4.2 \%$ of the children were registered with symptom of speech disorders. The early detection and diagnosis of environmental etiologic factors may be an important step to prevent malocclusions in children with primary dentition. Regular monitoring of the functional status allows timely orthodontic prevention and an early treatment according to each child's individual needs.
\end{abstract}

Keywords: orofacial dysfunctions, prevalence, primary dentition

\section{Introduction}

Today's emphasis on preventive orthodontic care necessitates rational planning of orthodontic preventive measures among children even in an early stage of dental development [1]. The functional matrix theory of growth and development by Moss [2] presumes that growth of the face occurs as a response to functional needs and neurotrophic influences, and is mediated by the soft tissues in which the jaws are embedded. In this conceptual view, the soft tissues grow, and both bone and cartilage react. The conclusion, based on Moss's theory, is that the correct functions of the orofacial complex-breathing, swallowing, and chewing - have a major influence on the growth and correct development of face, jaws, and dentition.

The last conducted study about the prevalence of malocclusions in primary dentition in Bulgaria was in the middle of the 80 's, where almost all of the children were in organized contingents and strictly examined by a dentist or an orthodontist every year. At that time an oral health prevention program was developed, which included orthodontic services for the masses [3,4]. After the change in health politics, this program is no longer active and occlusal characteristics, prevalence, and the types of malocclusions in the primary dentition are not regularly registered and analyzed. An optimal occlusion in primary dentition is essential for the further development of the occlusion in the permanent dentition [5.6]. The current preventive program for the Bulgarian children is mostly orientated to caries prevention but according to the literature, caries-reducing measures are not likely to have a significant influence on formation of malocclusions in primary dentition [7].

We need some present-day data about the prevalence of malocclusions in primary dentition and the aim of this study is to estimate dental health, prevalence of malocclusions and orofacial dysfunctions in a sample of 4-5-year-old Bulgarian kindergarten children. In this paper the prevalence of orofacial dysfunctions will be discussed.

\section{Materials And Methods}

The subjects were randomly selected from different kindergartens in the city of Plovdiv. Inclusion criteria were the existence of fully developed primary dentition, no orthodontic treatment, Caucasian origin, and a parental consent for participation in the study. A total of 471 boys and girls participated in this cross-sectional epidemiological study. The Ethics Committee of Medical University- Plovdiv approved the study (P-7781).

A postgraduate student in Orthodontics (KY) carried out the entire diagnostic assessment of all the children. A specific examination form was designed for this study which contains information about dental health, individual occlusion findings and functional status. All the findings were made under good lighting conditions. The functional examination entailed diagnosis of any orofacial dysfunctions, noteworthy extraoral findings and oral habits. The following parameters were evaluated:

2.1. Open mouth posture:

Lip incompetence

- $\quad$ Short everted upper lip 
- $\quad$ Distinct supramental fold

Impairment of nasal breathing was tested with cotton ball test. A cotton ball is placed in front of each nostril for 30 seconds by inviting the child to inhale and exhale as the opposite nostril closed with child's pointer. We distinguish between normal nasal breathing or impaired nasal breathing by the presence or absence of cotton ball's movements.

\subsection{Pathological tongue posture}

Kittel defines the physiological tongue posture [8] as the resting position of the tongue in contact with the palate extending to the palatal aspect of the alveolar ridge. We differentiated between pathological tongue postures as interdental tongue posture (tongue position between the anterior teeth) and caudal tongue posture (direct towards the lower anterior teeth).

\subsection{Swallowing patterns}

Physiological (somatic) swallowing is characterized by tongue contact with the hard palate [9] and the simultaneous absence of tongue contact with the anterior and canine teeth when swallowing while the lip and mentalis muscles are inactive. To diagnose the swallowing pat-tern, the children were asked to swallow saliva and water. Swallowing patterns were assigned into two categories:

- $\quad$ Physiological swallowing

- Infantile swallowing - tip of the tongue presses against or between the anterior or posterior teeth and activity of the orofacial muscles ("facial grimace")

\subsection{Habits}

Current habits were recorded individually by interviewing and clinically examining the sub-jects for signs of oral habits. In accordance with the examinations results, we added the following four repetitive habits to the checklist:

- $\quad$ Thumb-sucking

- $\quad$ Pacifier-sucking

- $\quad$ Lip-sucking

- $\quad$ Tongue-sucking

\subsection{Symptoms of speech disorders}

The children were asked to pronounce the following Bulgarian words "blue”, "rabbit” and „tractor" to examine them for speech disorder's symptoms. We used auditivy method for evaluating of the quality of pronunciation of the sounds "s", "z", "r".

2.6. Statistical methods: Collection, evaluation and a statistical analysis of the data were con-ducted using Microsoft ${ }^{\circledR}$ Excel and SPSS Version 17.0 for Windows ${ }^{\circledR}$ (SPSS Inc., Chicago, IL., USA). Means and standard deviations were determined as descriptive statistical values to characterize univariate frequency distributions of various variables. A comparison of absolute frequencies of specific characteristics was tested with Pearson's chi-square test. A statistical significance was assessed at the 5\% level.

\section{Results}

A total of 241 males and 230 females were examined "Fig. 1".
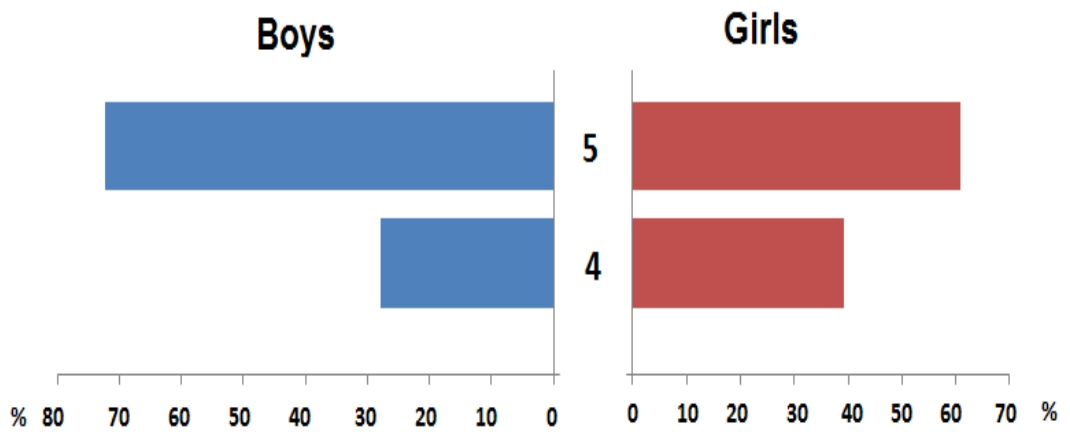

Age

Figure. 1. Age-gender pyramid of the sample 
Open mouth posture was observed in $46 \%$ of the children and the results for gender and age are shown on Table 1 and Table 2. There is no statistical difference between the age groups, but boys have shown slight increase in the frequency, however without statistical significance. Impaired nasal breathing was diagnosed in $19.5 \%$ of all participants. The slight differences between the age and gender groups is above the statistical significance level. Infantile swallowing pattern was observed in $36.7 \%$ of the children.

Table 1. Prevalence of incorrect orofacial functions by age

\begin{tabular}{|c|c|c|c|c|c|c|}
\hline \multirow[t]{3}{*}{ Incorrect orofacial functions } & \multicolumn{4}{|c|}{ Age } & \multirow{2}{*}{\multicolumn{2}{|c|}{ Total }} \\
\hline & \multicolumn{2}{|c|}{4 years olds } & \multicolumn{2}{|c|}{5 years olds } & & \\
\hline & n & $\%$ & n & $\%$ & $\bar{n}$ & $\%$ \\
\hline Impaired nasal breathing & 28 & 5.9 & 64 & 13.6 & 92 & 19.5 \\
\hline Infantile swallowing & 57 & 12.1 & 116 & 24.6 & 173 & 36.7 \\
\hline Open mouth posture & 72 & 15.3 & 145 & 30.8 & 217 & 46.1 \\
\hline
\end{tabular}

Table 2. Prevalence of incorrect orofacial functions by gender

\begin{tabular}{|c|c|c|c|c|c|c|}
\hline \multirow[t]{3}{*}{ Incorrect orofacial functions } & \multicolumn{4}{|c|}{ Gender } & \multicolumn{2}{|c|}{ Total } \\
\hline & \multicolumn{2}{|c|}{ Girls } & \multicolumn{2}{|c|}{ Boys } & & \\
\hline & $\mathbf{n}$ & $\%$ & $\mathbf{n}$ & $\%$ & $\mathbf{n}$ & $\%$ \\
\hline Impaired nasal breathing & 42 & 8.9 & 50 & 10.6 & 92 & 19.5 \\
\hline Infantile swallowing & 78 & 16.6 & 95 & 20.2 & 173 & 36.7 \\
\hline Open mouth posture & 97 & 20.6 & 120 & 25.5 & 217 & 46.1 \\
\hline
\end{tabular}

The prevalence between the age groups remains static and the males reports slight increase in frequency than females. The differences are not statistical significant. Pathological tongue posture was observed in $9 \%$ of all children. Interdental tongue posture is below 1\%, shown on Table 3 and Table 4, but appears for the first time in 5 years old group and the males group $\left(\chi^{2}=3,850 \mathrm{p}=.050\right)$.

Table 3. Prevalence of bad habits and incorrect tongue posture by age

\begin{tabular}{|c|c|c|c|c|c|c|}
\hline \multirow[t]{3}{*}{ Bad habit } & \multicolumn{4}{|c|}{ Age } & \multirow{2}{*}{\multicolumn{2}{|c|}{ Total }} \\
\hline & \multicolumn{2}{|c|}{4 years olds } & \multicolumn{2}{|c|}{5 years olds } & & \\
\hline & n & $\%$ & $\mathbf{n}$ & $\%$ & n & $\%$ \\
\hline Without sucking habit & 83 & 54,1 & 220 & 66,2 & 283 & 62,2 \\
\hline Lip sucking & 53 & 33.8 & 75 & 23.9 & 128 & 27,2 \\
\hline Finger sucking & 10 & 6.4 & 4 & 1.3 & 14 & 3,0 \\
\hline Pacifier sucking & 2 & 1.3 & 0 & 0 & 2 & 0,4 \\
\hline Tongue sucking & 0 & 0 & 2 & 0,6 & 2 & 0,4 \\
\hline Interdental tongue posture & 0 & 0 & 4 & 1.3 & 4 & 0,8 \\
\hline Caudal tongue posture & 9 & 5.7 & 29 & 9.2 & 38 & 8,1 \\
\hline
\end{tabular}

Caudal tongue posture was diagnosed in $8 \%$, where the females have slight increase in the prevalence than males, but no statistical differences were found. Near $60 \%$ of the children were registered without non nutrive sucking behavior. The most prevalent sucking habit is lip sucking with $27 \%$, followed by finger sucking with $3 \%$. Below $1 \%$ is the prevalence of pacifier-sucking and tongue-sucking in this sample. On Table 3 and 4 are shown gender and age associated prevalence of bad habits and incorrect tongue posture.

Table 4. Prevalence of bad habits and incorrect tongue posture by gender

\begin{tabular}{|c|c|c|c|c|c|c|}
\hline \multirow[t]{3}{*}{ Bad habit } & \multicolumn{4}{|c|}{ Gender } & \multirow{2}{*}{\multicolumn{2}{|c|}{ Total }} \\
\hline & \multicolumn{2}{|c|}{ Girls } & \multicolumn{2}{|c|}{ Boys } & & \\
\hline & $\mathbf{n}$ & $\%$ & $\mathbf{n}$ & $\%$ & $\mathbf{n}$ & $\%$ \\
\hline Without sucking habit & 142 & 50.2 & 141 & 49.8 & 283 & 60,1 \\
\hline Lip sucking & 67 & 14.2 & 61 & 13.0 & 128 & 27,2 \\
\hline Finger sucking & 4 & 0.8 & 10 & 2.1 & 14 & 3,0 \\
\hline Pacifier sucking & 0 & 0 & 2 & 0.4 & 2 & 0,4 \\
\hline Tongue sucking & 0 & 0 & 2 & 0,4 & 2 & 0,4 \\
\hline Interdental tongue posture & 0 & 0 & 4 & 0,8 & 4 & 0,8 \\
\hline Caudal tongue posture & 17 & 4,5 & 21 & 3,6 & 38 & 8,1 \\
\hline
\end{tabular}

The prevalence of finger-sucking $(\chi 2=9,423 \mathrm{p}=.002)$ and pacifier-sucking $(\chi 2=4,017 \mathrm{p}=.045)$ decreases with the age with statistical significance, while the prevalence of lip-sucking is increasing with the age with statistical significance $(\chi 2=5,155 \mathrm{p}=.023)$. The tongue-sucking habit appears for the first time in 5 years 
old group. Symptoms of speech disorders were diagnosed in $4.2 \%$ of the children in primary dentition. There were no gender-specific differences, but we found a tendency that more males were observed with these symptoms $(\chi 2=2,965 \mathrm{p}=.085)$. Additional consultation with speech disorders specialist is needed to confirm the diagnose.

\section{Discussion}

It has been shown by numerous investigators that bad habits, such as non-nutritive sucking habits, atypical swallowing, oral breathing, can be regarded as risk factors to develop malocclusions in the primary dentition [10, 11]. Research on the relationship between malocclusion and mouth breathing suggests that impaired nasal respiration may contribute to the development of increased facial height, anterior open bite, increased overjet, and narrow pal-ate, although it is not the sole or even the major cause of these conditions [11, 12]. Mouth breathing children often present with skeletal II discrepancies, with transverse maxillary constriction, increased anterior facial height, and obtuse mandibular-maxillary planes angle. These findings may relate to the inferior tongue posture and hypotonia of facial muscles associated with mouth breathing. $[11,12$, 13]. Larsson [14] reports a 70\% frequency of sucking in German population. The frequency we assessed of sucking habits in primary dentition can be considered low. Stahl et al. [15] reports 37\% frequency of open mouth posture in primary dentition, which is comparable with our results. They also report prevalence of caudal and interdental tongue posture $21.4 \%$ and $12.5 \%$, respectively, which is higher to our frequency rates. Ovsenik et al. [1] found 35\% prevalence of atypical swallowing pattern, while Stahl et al. [15] report 62\% frequency in primary dentition. It is difficult to compare this study's re-ported prevalence of orofacial dysfunctions and oral habits with results of other studies due to their different study designs, functional parameters and sample size. The lack of anamnestic data provided by parents or caregivers, short interviews and the subject's embarrassment had a significant impact on our oral-habit findings. We were unable to interview the parents beforehand, nor was their presence possible during the examination for this study.

\section{Conclusion}

The high frequency rates of orofacial dysfunctions and oral habits in primary dentition suggest their repetition in later dentitional stages. Functional factors disrupting dentitional development must be diagnosed and eliminated early. Moreover, children in primary dentition should undergo screening for malocclusions and orofacial dysfunctions. These must be treated with interdisciplinary measures when necessary [16]. Orthodontic prevention will always be an individual effort and can be successful only with the partnership of well-informed and convinced parents.

\section{References}

[1]. Ovsenik M, Farcnik FM,Verdenik I. Comparison of intra-oral and study cast measurements in the assessment of malocclusion. Eur J Orthod 2004 Jun;26(3):273-277.

[2]. Moss ML. The differential roles of periosteal and capsular functional matrices in orofacial growth. Eur J Orthod. 2007 May;29(suppl 1): i96-i101

[3]. Atanasov K. [Orthodontic prevention for the masses] [in Bulgarian] doctoral diss., Plovdiv, Bulgaria, 1989; p.8-10

[4]. Mutafchiev V, Dinkova M, Hranova V. [Distribution of malocclusions and their main etiologic factors in kindergarten children] [in Bulgarian] Stomatologiya (S) 1988; (3): 55-61

[5]. Kerosuo H. The role of prevention and simple interceptive measures in reducing the need for orthodontic treatment. Med Principles Pract. 2002; 11(suppl 1): 16-21.

[6]. Stahl F, Grabowski R. Orthodontic findings in the deciduous dentition and early mixed dentition - inferences for a preventive strategy. J Orofac Orthop 2003; 64(6): 401-416.

[7]. Stahl F, Grabowski R. Malocclusion and caries prevalence: is there a connection in the primary and mixed dentitions? Clin Oral Investig 2004; Jun 8(2): 86-90;

[8]. Kittel A. Myofunktionelle Therapie. (Schulz-Kirchner Verlag, 2014, p.18-25)

[9]. Garliner D. Myofunctional Therapy. (Philadelphia-London-Toronto: Saunders, 1976, p. 7)

[10]. Larsson E. Sucking, chewing and feeding habits and the development of crossbite: a longitudinal study of girls from birth to 3 years of age. Angle Orthod 2001; Apr 71(2):116-119.

[11]. American Academy of Pediatric Dentistry. Guideline on management of the developing dentition and occlusion in paediatric dentistry. Clinical Guidelines. 2014;36(6):251-261.

[12]. Fields Jr HW, Warren DW, Black B, Phillips CL. Relationship between vertical dentofacial morphology and respiration in adolescents. Am J Orthod Dentofacial Orthop. 1991;99(2):147-154.

[13]. Marcus CL, Brooks LJ, Draper KA, Gozal D, Halbower AC, Jones J, et al. Diagnosis and management of childhood obstructive sleep apnea syndrome. Pediatrics. 2012;130(3):714-755

[14]. Larsson E. Artificial sucking habits: Etiology, prevalence and effect on occlusion. Int J Orofacial Myology 1994; 20:10-21

[15]. Stahl F, Grabowski R, Gaebel M, Kundt G. Relationship between occlusal findings and orofacial myofunctional status in primary and mixed dentition. Part II: Prevalence of orofacial dysfunctions. J Orofac Orthop. 2007 Mar;68(2):74-90

[16]. Korbmacher HM, Schwan M. Berndsen S, et al. Evaluation of a new concept of myofunctional therapy in children. Int J Orofacial Myology 2004; Nov; 30:39-52 